\title{
Perfil dos usuários do serviço de teleatendimento sobre drogas de abuso VIVAVOZ
}

\author{
Profile of users of the VIVAVOZ telephone service on drugs of abuse
}

\author{
Marilise Fraga de Souza ${ }^{1}$, Eglê Rejane Kohlrausch ${ }^{2}$, Cláudia Galvão Mazoni ${ }^{3}$, Taís de Campos \\ Moreira $^{4}$, Simone Fernandes ${ }^{3}$, Denise Conceição Mesquita Dantas ${ }^{5}$, Maristela Ferigolo ${ }^{6}$, Helena \\ Maria Tannhauser Barros ${ }^{7}$
}

\begin{abstract}
${ }^{1}$ Enfermeira. Mestranda, Programa de Pós-graduação em Ciências Médicas, Universidade Federal de Ciências da Saúde de Porto Alegre (UFCSPA), Porto Alegre, RS. ${ }^{2}$ Enfermeira psiquiátrica. Mestre em Enfermagem, Universidade Federal do Rio Grande do Sul (UFRGS), Porto Alegre, RS. Professora de Saúde Mental, Escola de Enfermagem, UFRGS. ${ }^{3}$ Psicóloga. Mestre em Ciências Médicas, UFCSPA. Doutoranda em Ciências Médicas, UFCSPA. ${ }^{4}$ Fonoaudióloga. Mestre em Ciências Médicas, UFCSPA. Doutoranda em Ciências Médicas, UFCSPA. ${ }^{5}$ Farmacêutica. Doutora em Fisiologia, Universidade Estadual de Campinas (UNICAMP), Campinas, SP. Professora adjunta, Farmacologia, UFCSPA. ${ }^{6}$ Farmacêutica. Doutora em Fisiologia, UFRGS. Coordenadora, Serviço Nacional de Informações e Orientações sobre a Prevenção do Uso Indevido de Drogas - VIVAVOZ. ${ }^{7}$ Médica. Pós-doutorado em Neuropsicofarmacologia, Tufts University, Boston, USA. Professora titular, Farmacologia, UFCSPA.

Este estudo foi realizado na Universidade Federal de Ciências da Saúde de Porto Alegre (UFCSPA), Porto Alegre, RS, e no Serviço Nacional de Informações e Orientações sobre a Prevenção do Uso Indevido de Drogas - VIVAVOZ.

Apoio financeiro: Secretaria Nacional Antidrogas - Associação de Amparo à Pesquisa em Farmacologia e Toxicologia (SENAD/AAPEFATO), Coordenação de Aperfeiçoamento de Pessoal de Nível Superior (CAPES) e Conselho Nacional de Desenvolvimento Científico e Tecnológico (CNPq).
\end{abstract}

\section{Resumo}

Introdução: O abuso de drogas é um problema importante de saúde pública, e intervenções telefônicas são utilizadas como uma modalidade de tratamento. Objetivou-se descrever o perfil sociodemográfico, o padrão de consumo e a dependência de substâncias psicoativas dos usuários que buscaram auxílio em um serviço de teleatendimento para drogas de abuso.

Método: Foram utilizados os dados do $1^{\circ}$ ano de funcionamento do serviço, coletados por consultores previamente capacitados, através de protocolo informatizado. Foram aplicados instrumentos para caracterização sociodemográfica, padrão de consumo e dependência dos usuários. Utilizou-se estatística descritiva para estimar a distribuição das variáveis, e os dados apresentam-se em freqüências.

Resultados: No período, foram atendidas 28.257 ligações, sendo 7.956 incluídas no estudo. No total, foi encontrada maior prevalência de mulheres, estudantes, solteiros, maiores de 35 anos, com ensino fundamental incompleto e renda familiar menor que cinco salários mínimos procurando o teleatendimento. Dentre os usuários de drogas, predominaram homens, de 18 a 25 anos. As drogas mais utilizadas foram tabaco, maconha, álcool e cocaína. O uso de tabaco foi semelhante para ambos os sexos. Indivíduos do sexo masculino usaram mais drogas ilícitas. A maioria dos usuários era dependente, sendo que os homens apresentaram maiores índices de dependência para tabaco e solventes.

Conclusões: Esses resultados traçam o perfil do usuário que procura auxílio telefônico para a questão de drogas, e revelam a importância desses serviços à população, auxiliando no direcionamento de ações preventivas.

Descritores: Substâncias psicoativas, telemedicina, serviços de informação, epidemiologia.

\begin{abstract}
Introduction: Drug abuse is a major public health problem. Telephone interventions have been used as a treatment method. This study aimed at describing the sociodemographic profile, consumption pattern and dependence on psychoactive substances of individuals seeking help in a telephone service on drugs of abuse.
\end{abstract}

\footnotetext{
Correspondência:

Marilise Fraga de Souza, Rua Sarmento Leite, 245, Prédio Anexo 1, sala 206, CEP 90050-170, Porto Alegre, RS. Tel./Fax: (51) 3303.8825. E-mail: marifs@fffcmpa.edu.br 
Methods: Data were collected by previously trained consultants using an electronic protocol throughout the first year of the service. Instruments were applied to find the sociodemographic profile, consumption pattern and dependence of drug users. Descriptive statistics was used to estimate distribution of variables, and the data are presented as frequencies.

Results: Throughout the study period there were 28,257 calls, of which 7,956 were included. In total there was higher prevalence of women, students, single individuals, older than 35 years, with incomplete primary education and family income lower than five minimum wages. Men aged 18-25 years were prevalent in the sample. The most frequently used drugs were tobacco, cannabis, alcohol and cocaine. Tobacco use was similar for both genders. Males used more illicit drugs. Most drug users were dependent, and men had higher rates of addiction to tobacco and solvents.

Conclusions: These results outline the profile of individuals who seek care through a telephone service, showing the importance of these services for the population and guiding preventive actions.

Keywords: Psychoactive substances, telemedicine, information services, epidemiology.

\section{Introdução}

O abuso de drogas tornou-se um grande problema de saúde pública mundial. No Brasil, levantamentos sistemáticos têm sido realizados com o objetivo de estudar a prevalência do uso de álcool e outras drogas ${ }^{1-}$ ${ }^{6}$. No último Levantamento Domiciliar, realizado em 2005, o uso na vida de drogas ilícitas foi referido por $22,8 \%$ dos entrevistados, prevalecendo o uso de maconha $(8,8 \%)$ e solventes $(6,1 \%)$. O uso na vida de álcool e tabaco foi relatado, respectivamente, por $77,4 \%$ e $44 \%$ dos entrevistados ${ }^{2}$. Dados mais recentes sobre o consumo de álcool na população brasileira revelam que $52 \%$ dos indivíduos podem ser classificados como bebedores, e praticamente a metade desses (25\%) faz uso de álcool pelo menos uma vez por semana ${ }^{6}$. Além do crescente consumo, estudos apontam significativa diferença do uso de drogas entre os sexos. Indivíduos do sexo masculino, em geral, utilizam mais álcool ou outras drogas, revelando um importante padrão social do consumo dessas substâncias ${ }^{3,6-8}$.

O desenvolvimento da informática e das telecomunicações favoreceu o acesso a uma quantidade cada vez maior de pessoas a serviços de saúde à distância, viabilizando uma nova forma de assistência, a telemedicina ${ }^{9}$. Linhas telefônicas de informações, orientações e aconselhamento em saúde têm um importante papel dentro da telemedicina, possibilitando a troca de informações clínicas entre profissionais da saúde e pacientes ${ }^{10}$. Elas complementam as intervenções face a face e de auto-ajuda, além de serem úteis como estratégia de prevenção de recaída ao uso de drogas ${ }^{11}$. Segundo Lichenstein et al. ${ }^{10}$, o aconselhamento pode ser proativo, quando a ligação inicial é realizada pelo serviço telefônico ao usuário, e reativo, quando o usuário é quem procura o atendimento.

Dentre as vantagens apresentadas por essa técnica, salienta-se a obtenção de um serviço que atende na própria residência do cliente ou onde ele estiver. Além disso, são beneficiados também os indivíduos com limitações físicas, que moram em regiões onde não há acesso a tratamento ou que são relutantes em participar de intervenções face a face, muitas vezes decorrente da falta de tempo ou dificuldade financeira ${ }^{12,13}$.

Estudos internacionais mostram serviços de aconselhamento telefônico em muitas áreas da saúde, como pediatria ${ }^{14}$, cardiologia ${ }^{15}$, emergência ${ }^{16}$, sexologia ${ }^{17}$, cuidados paliativos a pacientes terminais ${ }^{18}$, psiquiatria ${ }^{19}$ e no tratamento da dependência química ${ }^{11,20-}$ ${ }^{22}$. No Brasil, os serviços telefônicos para informações sobre saúde também vêm sendo utilizados. Atualmente, a população brasileira conta com o Serviço Nacional de Informações e Orientações Sobre a Prevenção do Uso Indevido de Drogas, o VIVAVOZ, concebido pela Secretaria Nacional Antidrogas (SENAD) e pela Fundação Universidade Federal de Ciências da Saúde de Porto Alegre (UFCSPA). Esse serviço, que atende pelo número 0800-5100015, presta aconselhamento telefônico reativo, gratuito, anônimo, aberto à população em geral, e especializado em oferecer informações e orientações sobre drogas em linguagem adequada e desprovida de preconceito. Além do VIVAVOZ, existem outros serviços de teleatendimento no Brasil, entre eles: o Centro de Informações Toxicológicas do Rio Grande do Sul $(\mathrm{CIT} / \mathrm{RS})^{23}$, o Serviço de Informações sobre Substâncias Psicoativas (SISP) ${ }^{24}$, o Disque Pare de Fumar do Instituto Nacional do Câncer (INCA) ${ }^{25}$, o Serviço Nacional de Informações sobre Agentes Teratogênicos (SIAT) ${ }^{26}$, os Centro de Informações sobre Medicamentos (CIM $)^{27}$ e os Centros de Assistência e Informação Toxicológica (CEATOX ${ }^{28}$. Cada um desses serviços usa estratégias de contato de teleatendimento, além de prestar informações. O VIVAVOZ oferece informações científicas sobre drogas e disponibiliza Intervenção Breve Motivacional (IBM) ${ }^{29}$ para os usuários.

A participação de equipes de saúde na prevenção do uso de substâncias psicoativas na comunidade, no tratamento da dependência e na orientação a usuários ou familiares vem crescendo a cada $\mathrm{dia}^{30}$. Uma vez que esses profissionais têm contato direto com os usuários de drogas que buscam tratamento, informações atualizadas sobre as diferentes substâncias psicoativas são necessárias. Nesse sentido, é importante conhecer a situação de uso, abuso ou dependência de drogas no 
país, bem como o perfil dos usuários que procuram atendimento, para que se possa planejar e implementar ações que contemplem as reais necessidades da população.

Objetivou-se, portanto, descrever o perfil sociodemográfico, o padrão de consumo e a dependência de drogas dos usuários que buscaram auxílio telefônico em um serviço de informações e orientações sobre drogas.

\section{Método}

Trata-se de um estudo exploratório-descritivo retrospectivo, com abordagem quantitativa. Foi utilizado o banco de dados do serviço VIVAVOZ, gerado a partir de um software criado especificamente para o teleatendimento.

O VIVAVOZ recebeu, de 21 de junho de 2005 a 21 de junho de 2006, 172.274 chamadas, que compreendem as ligações discadas para o número
0800-510-0015 durante 24 horas do dia, todos os dias da semana. Dessas, 144.017 ligações não foram atendidas por terem sido realizadas fora do horário de atendimento (de segunda a sexta-feira, das $8 \mathrm{~h}$ às $20 \mathrm{~h}$ ) ou colocadas em espera para atendimento e não completadas. Os consultores do VIVAVOZ atenderam, nesse período, 28.257 ligações. Dessas, foram analisadas 7.956, que representaram a primeira ligação realizada pelo cliente, e que tiveram os dados completamente preenchidos. Foram excluídos os dados das ligações identificadas como retornos $(\mathrm{n}=1.686)$, e das ligações perdidas antes de serem registradas as informações sociodemográficas do cliente ou informações sobre o uso de drogas entre os usuários $(\mathrm{n}=18.615)$ (Figura 1). O registro incompleto das informações se deveu a vários fatores, como trotes, interrupção voluntária da ligação pelo cliente, queda da ligação durante o atendimento ou desistência do cliente em dar seguimento ao preenchimento dos protocolos. Nesses casos, foi respeitada a vontade do cliente e as informações coletadas não foram utilizadas.

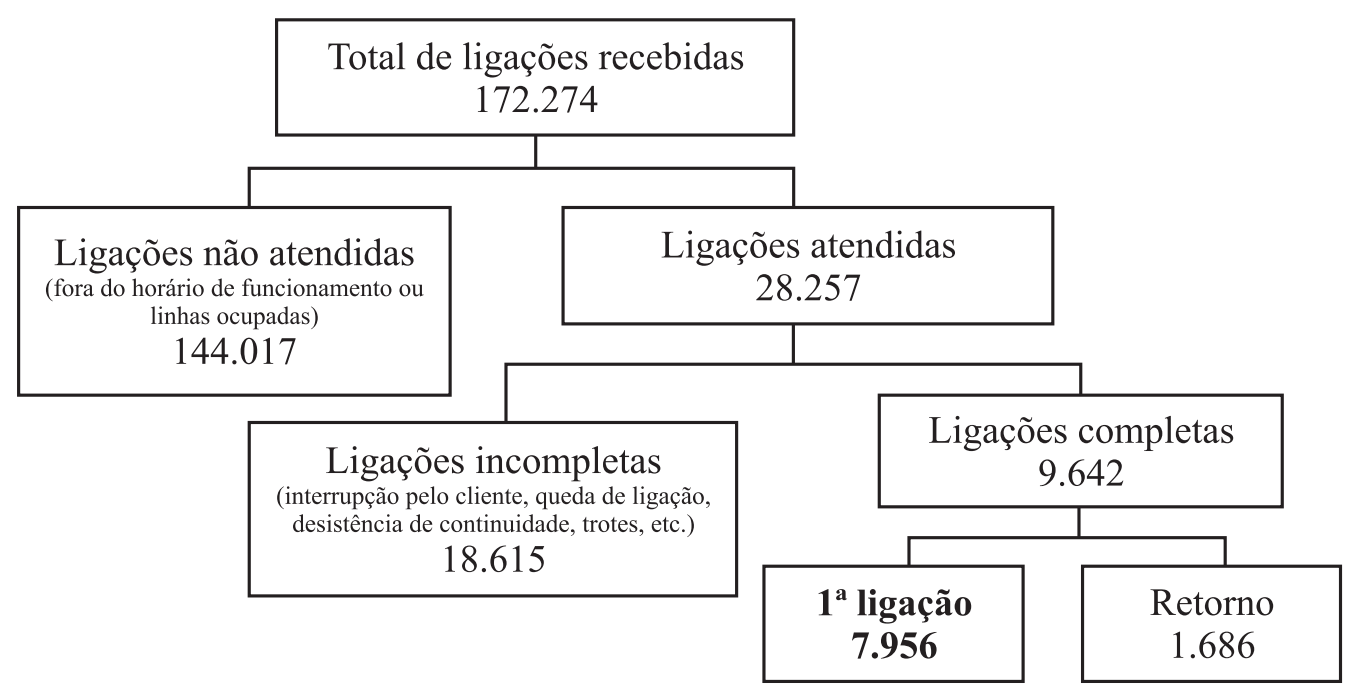

Figura 1 - Fluxograma das ligações recebidas pelo VIVAVOZ no primeiro ano de funcionamento

Os dados foram coletados por consultores capacitados para atendimento no call center e para aplicação da IBM aos usuários sob supervisão de profissionais da saúde. Os consultores foram submetidos a um processo de treinamento, composto de: a) aulas teóricas em formato de curso de extensão sobre as bases para abordagem da dependência química (40 horas); b) treinamento teórico-prático no call center para familiarização com o software (20 horas) e treinamento da entrevista motivacional e da IBM (20 horas); c) treinamento continuado com fins de manter a qualidade das informações prestadas. O modelo de treinamento utilizado foi adaptado do Medical Education Model for the Prevention and Treatment of Alcohol Use Disorders $^{31}$. Após esse processo, os consultores foram avaliados sistematicamente e supervisionados durante todo o tempo de atendimento no call center. $\mathrm{O}$ atendimento às ligações foi seguido de forma padrão a todos os clientes, através de um fluxograma ${ }^{9}$ baseado nos princípios da entrevista motivacional. 
O instrumento utilizado para registrar os dados sociodemográficos e os questionamentos dos clientes foi o protocolo geral de atendimento, que incluía informações sobre a classificação geral da pergunta (busca por centros de tratamento, material informativo, informações sobre drogas e outras) e caracterização do cliente (sexo, faixa etária, escolaridade, estado civil, profissão e renda familiar de quem buscava a informação). Adicionalmente, perguntas específicas sobre o padrão de consumo da substância utilizada (álcool, cocaína, maconha, solvente, tabaco e outras) foram aplicadas aos usuários de drogas. O questionário utilizado, proposto e desenvolvido pela Organização Mundial da Saúde (OMS) ${ }^{32}$, adaptado para o Brasil, e já utilizado em outros estudos multicêntricos ${ }^{1-3,33}$, contempla perguntas sobre a freqüência, quantidade, tempo e modo de uso das substâncias. De acordo com a OMS, o uso de drogas segue a seguinte classificação: uso na vida (uso pelo menos uma vez na vida), uso no ano (uso pelo menos uma vez nos últimos 12 meses), uso no mês (uso pelo menos uma vez nos últimos 30 dias) e uso pesado (uso diário no último mês) ${ }^{33}$.

Na seqüência, aplicou-se o questionário de avaliação de dependência do Substance Abuse and Mental Health Services Administration (SAMHSA) ${ }^{34}$, adaptado às condições do serviço. O usuário foi considerado dependente quando preencheu pelo menos dois dos seguintes critérios: no último ano gastou grande parte do tempo com a droga por 1 mês ou mais; usou drogas mais freqüentemente ou em quantidades maiores; necessitou de maiores quantidades da droga; esteve em situações de riscos físicos; teve algum problema pessoal pelo uso de drogas; quis diminuir ou parar o uso da droga ${ }^{34}$.

Para garantir os aspectos éticos neste estudo, foi aplicado verbalmente um termo de consentimento a todos os clientes, autorizando a utilização dos dados. O anonimato de quem usou o serviço telefônico foi garantido e o número de telefone de quem chamou não foi disponibilizado ao serviço, o que impediu de se completar o perfil das chamadas perdidas. O projeto foi aprovado pelo Comitê de Ética e Pesquisa da UFCSPA.

Os dados obtidos foram analisados com o programa SPSS 15.0. A estatística descritiva foi utilizada para estimar a distribuição das variáveis, que foram apresentadas em tabelas de freqüências absolutas e relativas. Aplicou-se o teste Qui-quadrado para comparação das características entre usuários e não usuários de drogas e da dependência entre os sexos. Foi considerado estatisticamente significativo $p<0,05$.

\section{Resultados}

No período do estudo, foram analisadas 7.956 ligações, sendo os usuários de drogas os que mais procuraram atendimento, com 2.600 ligações $(32,8 \%)$. Dentre os não usuários de drogas, destacam-se os parentes $(12,8 \%)$, amigos $(10,8 \%)$ e mães $(5,8 \%)$, os demais (pais, centros de tratamento, profissional de saúde e outros profissionais) totalizaram $37,8 \%$ das chamadas.

O perfil sociodemográfico dos não-usuários de drogas foi significativamente diferente do perfil dos usuários $(p<0,05)$, exceto em relação à renda familiar, que mostrou predominância de renda inferior a cinco salários mínimos para ambos os grupos. Para possibilitar uma melhor comparação, as características sóciodemográficas dos usuários e dos não usuários de drogas estão representadas na Tabela 1.

Analisando os motivos que levaram os clientes a procurarem o serviço, constatou-se que $64,2 \%$ buscavam informações sobre drogas, $28 \%$ solicitaram materiais informativos, $13,2 \%$, orientações sobre centros de tratamento e $13,2 \%$, outras informações. A maioria dos usuários de drogas $(63,2 \%)$ nunca havia procurado auxílio quanto aos problemas relacionados à situação que ocasionou a chamada telefônica.

Dentre as drogas mais questionadas pelos usuários do serviço, estão a maconha, a cocaína, o tabaco e o álcool. Por outro lado, entre os usuários, as drogas mais consumidas, na vida, no ano, no mês e diariamente, foram o tabaco, a maconha, o álcool, a cocaína e os solventes, respectivamente. A Tabela 2 apresenta a prevalência do consumo dessas drogas entre homens e mulheres, de acordo com as categorias de uso. Em geral, detecta-se maior porcentagem de uso de álcool e drogas ilícitas entre os homens, representando mais de $60 \%$ dos usuários de cada uma dessas substâncias. Para os usuários dessas drogas, vemos uma distribuição de 2 homens: 1 mulher. O tabaco é exceção, visto que há, praticamente, a proporção de 1 homem: 1 mulher.

Em relação ao tempo de uso das drogas, $68,5 \%$ dos usuários de tabaco, 67,8\% de álcool, 49,8\% de maconha e $35,2 \%$ de cocaína utilizavam a referida droga há mais de 5 anos, e 28,3\% dos usuários de solventes afirmaram ter menos de 6 meses de uso. Quanto à via de administração, 48,8\% dos usuários de cocaína a utilizavam na forma de pó, aspirada, e $45 \%$, inalado ou fumado, esta última caracterizando o uso do "crack".

Analisando as respostas de homens e mulheres em relação aos critérios de dependência de substâncias do SAMHSA $^{34}$, observou-se que uma parte dos usuários que ligaram já era dependente, sendo que os homens apresentavam significativamente mais dependência de tabaco ou solventes que as mulheres (Tabela 3 ). Detectou-se, portanto, que muitos usuários de drogas que ligaram ainda não apresentavam critérios suficientes para caracterizar possível dependência. Além disto, de todos os usuários (2.600), 24,7\% eram dependentes de tabaco. Tal relação foi de $10,4 \%$ de dependência para 
Tabela 1 - Características sociodemográficas da clientela do serviço $(n=7.956)$, dos usuários de drogas $(n=2.600)$ e dos não usuários de drogas $(\mathrm{n}=5.333)$ que efetuaram chamadas telefônicas ao VIVAVOZ, no período de 2005 a 2006

\begin{tabular}{|c|c|c|c|c|}
\hline & $\begin{array}{c}\text { Clientela do serviço, } \\
n *(\%)\end{array}$ & $\begin{array}{c}\text { Usuários de } \\
\text { drogas } n^{*}(\%)\end{array}$ & $\begin{array}{c}\text { Não usuários, } n^{*} \\
(\%)\end{array}$ & $\mathbf{p}$ \\
\hline \multicolumn{5}{|l|}{ Sexo } \\
\hline Masculino & $3.542(44,7)$ & $1.590(61,2)$ & $1.952(36,7)$ & \multirow{2}{*}{$<0,001^{\dagger}$} \\
\hline Feminino & $4.382(55,3)$ & $1.010(38,8)$ & $3.372(63,3)$ & \\
\hline \multicolumn{5}{|l|}{ Faixa etária } \\
\hline 12 a 17 anos & $1.327(22,5)$ & $309(15,1)$ & $1.017(26,0)$ & \multirow{4}{*}{$<0,001^{\dagger}$} \\
\hline 18 a 25 anos & $1.586(26,9)$ & $735(35,9)$ & $851(22,0)$ & \\
\hline 26 a 34 anos & $1.110(18,8)$ & $435(21,2)$ & $672(17,0)$ & \\
\hline$>35$ anos & $1.878(31,8)$ & $569(27,8)$ & $1.307(35,0)$ & \\
\hline \multicolumn{5}{|l|}{ Escolaridade } \\
\hline Analfabeto & $16(0,3)$ & $9(0,6)$ & $7(0,2)$ & \multirow{7}{*}{$<0,001^{\dagger}$} \\
\hline Ensino fundamental incompleto & $1.697(35,0)$ & $468(31,0)$ & $1.229(36,7)$ & \\
\hline Ensino médio incompleto & $951(19,6)$ & $280(19,0)$ & $671(20,0)$ & \\
\hline Curso técnico & $83(1,7)$ & $22(1,5)$ & $61(1,8)$ & \\
\hline Ensino fundamental completo & $538(11,1)$ & $222(15,0)$ & $316(9,4)$ & \\
\hline Ensino médio completo & $1.108(22,9)$ & $378(25,0)$ & $730(21,8)$ & \\
\hline Superior incompleto & $455(9,4)$ & $118(7,9)$ & $337(10,1)$ & \\
\hline \multicolumn{5}{|l|}{ Estado civil } \\
\hline Solteiro & $1.999(56,1)$ & $972(56,0)$ & $2.295(56,4)$ & \multirow{4}{*}{$<0,001^{\dagger}$} \\
\hline Casado & $458(34,3)$ & $588(34,0)$ & $1.411(34,7)$ & \\
\hline Separado & $3.267(7,9)$ & $172(9,8)$ & $286(7,0)$ & \\
\hline Viúvo & $98(1,7)$ & $18(1,0)$ & $80(2,0)$ & \\
\hline \multicolumn{5}{|l|}{ Profissão } \\
\hline Aposentado & $171(2,9)$ & $74(4,2)$ & $97(2,4)$ & \multirow{7}{*}{$<0,001^{\dagger}$} \\
\hline Autônomo & $207(3,5)$ & $94(5,4)$ & $113(2,7)$ & \\
\hline Desempregado & $378(6,4)$ & $226(12,9)$ & $152(3,7)$ & \\
\hline Do lar & $448(7,6)$ & $127(7,3)$ & $321(7,8)$ & \\
\hline Estudante & $1.887(32,1)$ & $363(20,8)$ & $1.524(36,9)$ & \\
\hline Profissional da saúde & $158(2,7)$ & $22(1,3)$ & $136(3,3)$ & \\
\hline Profissional de outras áreas & $2.624(44,7)$ & $841(48,1)$ & $1.783(43,2)$ & \\
\hline \multicolumn{5}{|l|}{ Renda familiar } \\
\hline 1 a 5 salários mínimos & $4.174(81,6)$ & $1.234 \quad(83,0)$ & $2.940(81,2)$ & \multirow{3}{*}{ NS } \\
\hline 5 a 10 salários mínimos & $672(13,1)$ & $179(12,0)$ & $493(13,6)$ & \\
\hline Mais de 10 salários mínimos & $267(5,2)$ & $78(5,2)$ & $189(5,2)$ & \\
\hline
\end{tabular}

* O número da amostra para cada critério avaliado variou de acordo com as respostas dos clientes.

$\uparrow$ Representa significância estatística no teste do qui-quadrado $(\mathrm{p}<0,05)$.

álcool, 12,1\% para maconha, 7,0\% para cocaína e 0,7 $\%$ para solventes.

\section{Discussão}

A caracterização da clientela do serviço nacional revelou maioria das ligações realizadas por indivíduos do sexo feminino, estudantes, solteiros, maiores de 35 anos, com ensino fundamental incompleto e renda familiar baixa, e a busca por informações sobre drogas de abuso como a maior demanda de ligações, sendo a cocaína e a maconha as mais questionadas. Esses dados são semelhantes aos obtidos há vários anos por outro serviço brasileiro de projeção regional, referente a informações sobre substâncias psicoativas ${ }^{24}$.

A predominância de mulheres na busca por serviços de saúde já é bastante evidenciada ${ }^{35,36}$. Quando se trata de serviços de atendimento para a questão das drogas, tal predomínio pode ser justificado pela influência da 
Tabela 2 - Prevalência do consumo de drogas entre homens e mulheres usuários de drogas $(\mathrm{n}=2.600)$ que procuraram o serviço VIVAVOZ, no período de 2005 a 2006, segundo os critérios do SAMHSA

\begin{tabular}{|c|c|c|c|c|}
\hline Droga*/Sexo & Uso na vida ${ }^{\dagger}, \mathrm{n}(\%)$ & Uso no ano ${ }^{\ddagger}, \mathrm{n}(\%)$ & Uso no mês", n (\%) & Uso pesado, n (\%) \\
\hline \multicolumn{5}{|c|}{ Tabaco $(\mathrm{n}=1.706)$} \\
\hline Masculino & $854(50,1)$ & $808(50,7)$ & $804(50,6)$ & $784(50,9)$ \\
\hline Feminino & $852(49,9)$ & $785(49,3)$ & $786(49,4)$ & $755(49,1)$ \\
\hline \multicolumn{5}{|c|}{ Maconha $(n=910)$} \\
\hline Masculino & $620(68,1)$ & $578(67,8)$ & $569(68,1)$ & $412(70,5)$ \\
\hline Feminino & $290(31,9)$ & $275(32,2)$ & $266(31,9)$ & $172(29,5)$ \\
\hline \multicolumn{5}{|l|}{ Álcool $(\mathrm{n}=828)$} \\
\hline Masculino & $557(67,3)$ & $528(67)$ & $509(66,5)$ & $162(67,2)$ \\
\hline Feminino & $271(32,7)$ & $260(33)$ & $256(33,5)$ & $79(32,8)$ \\
\hline \multicolumn{5}{|l|}{ Cocaína $(n=814)$} \\
\hline Masculino & $556(68,3)$ & $531(69,1)$ & $504(68,8)$ & $214(70,9)$ \\
\hline Feminino & $258(31,7)$ & $237(30,9)$ & $229(31,2)$ & $88(29,1)$ \\
\hline \multicolumn{5}{|l|}{ Solvente $(n=60)$} \\
\hline Masculino & $37(61,7)$ & $33(58,9)$ & $30(60)$ & $13(56,5)$ \\
\hline Feminino & $23(38,3)$ & $23(41,1)$ & $20(40)$ & $10(43,5)$ \\
\hline
\end{tabular}

\footnotetext{
* O número da amostra para cada droga avaliada varia de acordo com as respostas dos clientes.

† Porcentagem em relação a todos os indivíduos que usaram a droga ao menos uma vez na vida.

* Porcentagem em relação a todos os indivíduos que usaram a droga ao menos uma vez no último ano.

" Porcentagem em relação a todos os indivíduos que usaram a droga ao menos uma vez no último mês.

『 Porcentagem em relação a todos os indivíduos que usaram a droga diariamente no último mês (uso pesado).
}

família e sua responsabilidade na socialização dos filhos ${ }^{37}$, levando em conta que os diferentes levantamentos nacionais demonstram que as mulheres não costumam ser as maiores usuárias dessas substâncias ${ }^{3,6-8}$. O papel da mãe começa logo no início da gravidez, ao assumir a responsabilidade pelos cuidados infantis. As mulheres vêem os seus filhos como uma extensão de si próprias, dedicando-se a eles e sentindo-se responsáveis pelos seus atos ${ }^{37}$. Além disso, as mulheres parecem ser mais afetadas pela disfunção familiar devido ao consumo de substâncias psicoativas, sendo muitas vezes vítimas de violência pelos companheiros ou, até mesmo, pelos próprios filhos ${ }^{38}$, já que o uso de drogas começa cada vez mais cedo e os adolescentes copiam os modelos dos adultos também na freqüência do consumo de drogas ${ }^{39}$. Todas essas associações justificam a maior procura pelas mulheres a atendimento relacionado a drogas.

A constatação de uma maior demanda de esclarecimentos sobre drogas de abuso pode também demonstrar uma carência de serviços especializados em prestar essas informações, uma vez que eles são pouco comuns no Brasil ${ }^{9}$. O fato de a população brasileira ter procurado mais informações sobre cocaína e maconha, em detrimento daquelas sobre álcool e tabaco, como seria esperado pela importância epidemiológica desses, pode ser explicado pelo fato de estes últimos serem substâncias lícitas e, ainda, não consideradas drogas por muitos indivíduos. Por outro lado, a veiculação na mídia dos prejuízos relacionados ao uso de álcool e tabaco está cada vez mais freqüente. Porém, informações sobre as drogas ilícitas são mais escassas. Nesse contexto, drogas lícitas e ilícitas passam a ser encaradas de modo distinto pela opinião pública, gerando posturas extremamente incoerentes sob a ótica da saúde ${ }^{40}$. Embora a OMS considere que a eficiência da comunicação, da cooperação e da troca de informações e de experiências é essencial para a prevenção do uso de drogas ${ }^{41}$, e que é inegável a importância do papel da mídia na sociedade, intervenções preventivas puramente informativas sobre o uso de drogas são limitadas, pois, quando realizadas de maneira irresponsável, podem até mesmo influenciar o aumento do seu consumo ${ }^{40}$.

$\mathrm{O}$ acesso da população com menor índice educacional e poder aquisitivo ao atendimento referente a questões sobre drogas reflete a utilidade pública dos serviços de teleatendimento gratuitos e de fácil acesso aos seus usuários. O telefone é uma forma de comunicação que favorece esclarecimentos sobre questões que dificilmente seriam expostas se o contato fosse face a face. Além disso, é importante, do ponto de vista terapêutico, o caráter motivador do serviço, que auxilia usuários de drogas na procura por tratamento especializado $^{24}$. O maior número de clientes que buscaram auxílio no VIVAVOZ referiu baixo grau de escolaridade e baixa renda familiar, não dispondo de 
Tabela 3 - Prevalência de dependentes entre homens e mulheres usuários de drogas $(n=2.600)$ que procuraram o serviço VIVAVOZ, no período de 2005 a 2006, segundo os critérios do SAMHSA

\begin{tabular}{cccc}
\hline \multirow{2}{*}{ Droga*/Sexo } & \multicolumn{2}{c}{ Dependentes } & p \\
\cline { 2 - 3 } & $\mathbf{n}$ & $\%^{\dagger}$ & \\
\hline Tabaco (n=1.706) & 514 & 59,1 & \\
Masculino (869) & 437 & 50,8 & $<0,001^{\ddagger}$ \\
Feminino (860) & & & \\
Maconha (n=910) & 323 & 52,1 & 0,257 \\
Masculino (620) & 143 & 49,3 & \\
Feminino (290) & & & \\
Álcool (n=828) & 268 & 48,1 & 0,515 \\
Masculino (557) & 132 & 48,7 & \\
Feminino (271) & & & \\
Cocaína (n=814) & 185 & 33,3 & 0,502 \\
Masculino (556) & 85 & 32,9 & \\
Feminino (258) & & & \\
Solvente (n=60) & 21 & 56,8 & $0,042^{\ddagger}$ \\
Masculino (37) & 7 & 30,4 & \\
Feminino (23) & & & \\
\hline
\end{tabular}

* O número da amostra para cada droga avaliada varia de acordo com as respostas dos clientes.

† Porcentagem em relação ao número de usuários que usaram a droga na vida

* Representa significância estatística no teste do qui-quadrado $(\mathrm{p}<0,05)$.

condições para buscar tratamentos especializados ou particulares, devido ao seu alto custo. No entanto, devese considerar que o uso de drogas não é exclusividade de determinada classe socioeconômica, distribuindo-se regularmente por todas elas ${ }^{3}$.

Por outro lado, embora o problema da dependência química seja amplamente distribuído e discutido em todos os níveis da sociedade, o preconceito e a discriminação ainda estão entre os principais obstáculos ao tratamento e aos cuidados de pessoas com farmacodependências e problemas associado ${ }^{42}$. $\mathrm{O}$ fato de o VIVAVOZ ser um serviço anônimo, gratuito e passível de ser acessado de qualquer local, pode justificar o grande número de indivíduos que nunca havia procurado auxílio para os problemas relacionados ao consumo de drogas, e que optaram por esse aconselhamento telefônico. Assim, o telefone pode ser visto como uma alternativa para potencializar a efetividade das intervenções clínicas e a qualidade do atendimento aos pacientes ${ }^{43}$.

A maior porcentagem dos indivíduos que buscaram atendimento no serviço era de usuários de drogas e, diferentemente do restante da clientela do VIVAVOZ, predominantemente homens na faixa etária de 18 a 25 anos. Também foi constatado um significativo padrão de uso e um alto índice de dependência às drogas lícitas e ilícitas, com diferenças entre os sexos para algumas substâncias. Provavelmente por ser um serviço já dirigido à população de usuários de drogas, a prevalência desses entre os que buscaram auxílio telefônico no VIVAVOZ foi mais elevada que a encontrada em outro serviço telefônico de informações sobre substâncias psicoativas $^{24}$, refletindo uma sensibilização da população pretendida pelo serviço. De modo semelhante, dados de um serviço brasileiro de atendimento face a face para usuários de $\operatorname{drogas}^{44}$, e de um serviço telefônico de informações sobre doping da Suécia $^{22}$ também mostram o consumo de drogas mais intenso em indivíduos do sexo masculino e entre os adultos jovens. Também a dependência para drogas ocorre mais freqüentemente nessa faixa etária, e em menor intensidade após os 45 anos de idade 7 .

Estudos que caracterizem a clientela de serviços telefônicos reativos de informações sobre drogas de abuso são escassos. Entretanto, estudos internacionais que avaliam a eficácia desse tipo de programa têm sido realizados e revelam que dependentes químicos podem permanecer várias semanas consecutivas em abstinência, através da intervenção com as linhas telefônicas de ajuda ${ }^{45-47}$. Também há demonstração de que os pacientes podem requerer cuidados menos intensivos do que após intervenções do tipo face a face ${ }^{48}$. Adicionalmente, pacientes que recebem intervenção telefônica apresentam maior probabilidade de dar 
seguimento ao tratamento da dependência química do que aqueles que não recebem intervenção ${ }^{49}$, sendo que as mulheres apresentam maiores índices de continuidade desse tipo de tratamento ${ }^{50}$. A avaliação da efetividade das linhas telefônicas de ajuda sobre drogas mostra que mais de $40 \%$ das informações fornecidas pelos call centers sobre álcool, cocaína e heroína foram consideradas úteis pelos clientes ${ }^{51}$. Com isso, há relevância no atendimento telefônico para usuários e não-usuários de drogas que procuram por apoio, informação ou auxílio efetivo para a cessação do uso de drogas. Demonstra-se que, no caso do Brasil, essas formas de apoio são aplicáveis e socialmente úteis ao atingirem populações de mais baixo nível socioeconômico.

Em síntese, apesar de alguns usuários desejarem o abandono do uso de drogas ${ }^{1}$, de, eventualmente, fazerem tentativas, poucos são aqueles que têm sucesso ${ }^{52}$. Os motivos para tanto incluem vários fatores, inclusive a falta de algum tipo de suporte para atingirem os objetivos. Nesse sentido, as intervenções telefônicas podem ser úteis para oferecer suporte na tentativa do abandono do uso de drogas ou evitar recaídas ${ }^{10,53}$.

Os resultados relacionados ao consumo das drogas mais utilizadas pela clientela do VIVAVOZ mostram que o perfil de freqüência de consumo das várias drogas, entre usuários que ligam para o serviço de teleatendimento, é diferente do perfil de estudos epidemiológicos do Brasil ${ }^{1-5,54}$ e de outros países ${ }^{8,34}$, conforme já discutido anteriormente para a busca de informações. Com isso, houve um número proporcionalmente maior de usuários de drogas procurando por atendimento e, dentre tais usuários, uma proporção maior de dependentes do que o encontrado na população geral ${ }^{1,2}$. Os dados deste estudo revelam que, aproximadamente, metade dos usuários dessas drogas foi classificada como dependente, com exceção da cocaína, em que a proporção de dependentes era menor, em torno de $30 \%$. Houve maior proporção de dependentes homens para o tabaco e solventes, mostrando que o sexo feminino busca mais atendimento para as dependências que o masculino, à semelhança do que ocorre para outros problemas de saúde ${ }^{35}$, visto que as mulheres costumam ter uma representatividade populacional para dependência menor que os homens ${ }^{55}$.

Com os resultados deste estudo, se traçou o perfil da clientela de um serviço telefônico para o uso e abuso de drogas lícitas e ilícitas, que tem alcance nacional, descrevendo proporções de usuários que fazem consumo e que são dependentes. Esses dados são de relevância aos profissionais de saúde, já que conhecer a realidade do uso de drogas possibilita saber para quais substâncias a prevenção deve ser enfatizada, qual a droga ideal de se começar as atividades de prevenção, qual o sexo mais propenso a usar determinadas drogas e a influência do nível socioeconômico no uso, além de permitir planejamento por parte dos serviços de saúde para o número de pacientes que procuram tratamento para cada tipo de droga. Além disso, os dados revelam que esse serviço de teleatendimento é mais uma alternativa para suprir a carência de informações sobre drogas, podendo complementar o apoio aos pacientes em intervenções face a face de forma individualizada ou motivando indivíduos para a mudança do seu comportamento-problema e busca de auxílio adicional para o uso problemático da droga. Conhecer as características sociodemográficas de quem procura ajuda também pode ser importante no planejamento das ações de saúde, na medida em que fornecem dados sobre as necessidades da população quanto aos reais problemas relacionados às diferentes substâncias de abuso $^{56}$.

Os resultados deste estudo são limitados, pois não representam o perfil epidemiológico da população brasileira, mas dos usuários de um serviço de atendimento aos problemas relacionados ao uso de drogas. Certamente, a melhor comparação para esse grupo de indivíduos que buscam atendimento telefônico seria aquela com pessoas que procuram outros serviços telefônicos para atendimento de saúde. No entanto, ainda não dispomos de outros serviços de teleatendimento a nível nacional. Em vista disso, a discussão do consumo de drogas foi baseada em levantamentos epidemiológicos existentes no Brasil e em outros países. Além disso, os instrumentos utilizados para coleta das informações são baseados nas respostas dos clientes, sem confirmação por exames laboratoriais. A extensão das conclusões também só pode ser aplicada aos clientes que finalizaram a entrevista, e os dados podem diferir para os que estabeleceram um contato incompleto com o serviço. Estudos futuros devem ser planejados para lidar com cada uma dessas questões. Portanto, a continuidade de pesquisas sobre o tema drogas e alternativas de intervenções ao tratamento da dependência química é muito relevante, pois a informação pode ser uma importante ferramenta para diminuir os índices de uso, abuso e dependência de substâncias psicoativas.

\section{Agradecimentos}

SENAD/AAPEFATO oferecem o apoio financeiro para o funcionamento e para as pesquisas desenvolvidas no VIVAVOZ, além das bolsas de mestrado (T.C.M., S.F.) e doutorado (C.G.M.). Reconhece-se também o apoio do $\mathrm{CNPq}$, através de bolsa de produtividade de pesquisa $1 \mathrm{C}$ (H.M.T.B.).

Agradecimento aos consultores VIVAVOZ. 


\section{Referências}

1. Carlini EA, Galduróz JC, Noto AR, Nappo SA. I Levantamento domiciliar sobre o uso de drogas psicotrópicas no Brasil: estudo envolvendo as 107 maiores cidades do país - 2001. São Paulo: Centro Brasileiro de Informações Sobre Drogas Psicotrópicas (CEBRID); 2002.

2. Carlini EA, Galduróz JCF, Noto AR, Fonseca AM, Carlini CMA, Oliveira LG, et al. II Levantamento domiciliar sobre o uso de drogas psicotrópicas no Brasil: estudo envolvendo as 108 maiores cidades do país - 2005. Brasília: Secretaria Nacional Antidrogas; 2007.

3. Galduróz JC, Noto AR, Nappo SA, Carlini EA. Trends in drug use among students in Brazil: analysis of four surveys in 1987, 1989, 1993 and 1997. Braz J Med Biol Res. 2004;37(4):523-31.

4. Galduróz JCF, Noto AR, Fonseca AM, Carlini EA. V Levantamento Nacional Sobre o Consumo de Drogas Psicotrópicas entre estudantes do ensino fundamental e médio da rede pública de ensino nas 27 capitais brasileiras - 2004. São Paulo: Centro Brasileiro de Informações Sobre Drogas Psicotrópicas; 2005.

5. Galduróz JC, Noto AR, Nappo SA, Carlini EA. Household survey on drug abuse in Brazil: Study involving the 107 major cities of the country-2001. Addict Behav. 2005;30(3):545-56.

6. Laranjeira R, Pinsky I, Zaleski M, Caetano R. I levantamento nacional sobre os padrões de consumo de álcool na população brasileira. Brasília: Secretaria Nacional Antidrogas; 2007.

7. Ferigolo M. Uso de drogas em indivíduos institucionalizados e associação entre fatores de risco e dependência de drogas ilícitas [tese]. Porto Alegre: UFRGS; 2004.

8. Observatório Europeu da Droga e da Toxicodependência (OEDT). Relatório Anual 2005: a evolução do fenômeno da droga na europa. Luxemburgo: Serviço das Publicações Oficiais das Comunidades Européias; 2005.

9. Mazoni CG, Bisch NK, Freese L, Ferigolo M, Barros H. Aconselhamento telefônico reativo para cessação do consumo do tabaco: relato de caso. Aletheia. 2006;24:137-48.

10. Lichtenstein E, Glasgow RE, Lando HA, Ossip-Klein DJ, Boles SM. Telephone counselling for smoking cessation: rationales and meta-analytic review of evidence. Health Educ Res. 1996;11(2):243-57.

11. Wakefield M, Borland R. Saved by the bell: the role of telephone helpline services in the context of mass-media anti-smoking campaigns. Tob Control. 2000;9(2):117-54.

12. Pressman S, Carneiro E, Gigliotti A. Tratamentos nãofarmacológicos para o tabagismo. Rev Psiquiatr Clin. 2005;32(5):267-75.

13. Zhu SH, Tedeschi GJ, Anderson CM, Pirce JP. Telephone counseling for smoking cessation: what's in a call? J Couns Dev. 1996;75:93-102.

14. Bolli S, Melle GV, Laubscher B. After-hours paediatric telephone triage and advice: the Neuchâtel experience. Eur J Pediatr. 2005;164(9):568-72.

15. Cleland JG, Louis AA, Rigby AS, Janssens U, Balk AH; TEN-HMS Investigators. Noninvasive home telemonitoring for patients with heart failure at high risk of recurrent admission and death: the Trans-European Network-Home-Care Management System (TENHMS) study. J Am Coll Cardiol. 2005;45(10):1654-64

16. Lamkin S, Pohler HF. Ring ring..."Hello, drug and poison”. J Emerg Nurs. 2007;33(5):509-10.

17. Fugl-Meyer KS, Arrhult H, Pharmanson H, Bäckman AC, FuglMeyer AM, Fugl-Meyer AR. A Swedish telephone help-line for sexual problems: a 5-year survey. J Sex Med. 2004;1(3):278-83.

18. Lloyd-Williams M, Rashid A. An analysis of calls to an out-ofhours palliative care advice line. Public Health. 2003;117(2):125-7.

19. Crooks VC, Clark L, Petitti DB, Chui H, Chiu V. Validation of multi-stage telephone-based identification of cognitive impairment and dementia. BMC Neurol. 2005;5(1):8.

20. Parker JD, Turk CL, Busby LD. A brief telephone intervention targeting treatment engagement from a substance abuse program wait list. J Behav Health Serv Res. 2002;29(3):288-303.

21. McKay JR, Lynch KG, Shepard DS, Ratichek S, Morrison R, Koppenhaver J, et al. The effectiveness of telephone-based continuing care in the clinical management of alcohol and cocaine use disorders: 12-month outcomes. J Consult Clin Psychol. 2004;72(6):967-79.

22. Eklöf AC, Thurelius AM, Garle M, Rane A, Sjöqvist F. The antidoping hot-line, a means to capture the abuse of doping agents in the Swedish society and a new service function in clinical pharmacology. Eur J Clin Pharmacol. 2003;59(8-9):571-7.

23. Centro de Informações Toxicológicas. O que é o CIT. http:// www.cit.rs.gov.br. Acessado 06 jun 2008.

24. Ferigolo M, Gomez R, Rhoden CR, Malysz AS, Arbo E, Barros HMT. Informações sobre substâncias psicoativas por telefone: análise de dez anos de atividades. Rev AMRIGS. 2002;46(3-4):123-8.

25. Instituto Nacional do Câncer. Tabagismo. http://www.inca.gov.br/ tabagismo. Acessado 06 jun 2008.

26. Serviço Nacional de Informações sobre Agentes Teratogênicos. O que é o SIAT? http://www.hupes.ufba.br/siat/siat bahia .htm. Acessado 06 jun 2008

27. Silva CDC, Coelho HLL, Arrais PSD, Cabral FR. Centro de informação sobre medicamentos: contribuição para o uso racional de fármacos. Cad. Saude Publ. 1997;13(3):531-5.

28. Gandolfi E, Andrade MG. Eventos toxicológicos relacionados a medicamentos no Estado de São Paulo. Rev Saude Publica. 2006;40(6):1056-64.

29. Miller WR, Rollnick S. Entrevista motivacional: preparando as pessoas para a mudança de comportamentos adictivos. Porto Alegre: Artmed. 2001

30. Spricigo JS, Alencastre MB. O enfermeiro na unidade básica de saúde e os usuários de drogas: um estudo de Biguaçu - SC. Rev Latino-Am Enfermagem. 2004;12:427-32.

31. Murray M, Fleming M. Prevention and treatment of alcohol-related problems: an international medical education model. Acad Med. 1996;71(11):1204-10.

32. Smart RG, Arif A, Hughies P, Mora ME, Navaratnam V, Varma VK, et al. Drug use among non-student youth. WHO offset publication 60. Genebra: World Health Organization; 1981.

33. Galduróz JCF, Noto AR, Carlini EA. IV Levantamento sobre uso de drogas entre estudantes de $1^{\circ}$ e $2^{\circ}$ graus em 10 Capitais Brasileiras: 1997. São Paulo: Centro Brasileiro de Informações Sobre Drogas Psicotrópicas (CEBRID), UNIFESP; 1997.

34. Substance Abuse and Mental Health Services Administration (SAMHSA). Results from the 2001 national household survey on drug abuse. Rockville: SAMHSA. 2002.

35. Gomes R, Nascimento EF, Araújo FC. Por que os homens buscam menos os serviços de saúde do que as mulheres? As explicações de homens com baixa escolaridade e homens com ensino superior. Cad. Saude Publica. 2007;23(3):565-74.

36. Travassos C, Viacava F, Pinheiro R, Brito A. Utilização dos serviços de saúde no Brasil: gênero, características familiares e condição social. Rev Panam Salud Publica. 2002;11(5-6):365-73.

37. Roldan MCB, Galera SAF. Perception of the mothering role of women who live in a context of drugs and violence. Rev Latino-Am Enfermagem. 2005;13(2 n. esp):1118-26.

38. Rabello PM, Caldas Júnior AF. Violência contra a mulher, coesão familiar e drogas. Rev Saude Publica. 2007;41(6):970-8.

39. Njaine K, Minayo MCS. A violência na mídia como tema da área da saúde pública: revisão da literatura. Cienc Saude Coletiva. 2004;9(1):201-11.

40. Noto AR, Baptista MC, Faria ST, Nappo AS, Galduróz JCF, Carlini EA. Drogas e saúde na imprensa brasileira: uma análise de artigos publicados em jornais e revistas. Cad Saude Publica. 2003;19(1):6979.

41. World Health Organization (WHO). Outcome evaluation summary report: WHO/UNODC global initiative (1999-2003) on primary prevention of substance abuse. Genebra: WHO; 2007.

42. Organização Mundial da Saúde (OMS). Neurociências: consumo e dependência de substâncias psicoativas: resumo. Genebra: OMS, 2004

43. Friedman RH, Stollerman JE, Mahoney DM, Rozenblyum L. The virtual visit: using telecommunications technology to take care of patients. J Am Med Inform Assoc. 1997;4(6):413-25.

44. Passos SRL, Camacho LAB. Características da clientela de um centro de tratamento para dependência de drogas. Rev Saude Publica. 1998;32(1):64-71. 
45. Carroll KM, Rounsaville BJ, Nich C, Gordon LT, Wirtz PW, Gawin F. One-year follow-up of psychoteraphy and pharmacotheraphy for cocaine dependence. Delayed emergence of psychotherapy effects. Arch Gen Psychiatry. 1994;51(12):989-97.

46. Higgins ST, Wong CJ, Badger GJ, Ogden DE, Dantona RL. Contingent reinforcement increases cocaine abstinence during outpatient treatment and 1 year of follow-up. J Consult Clin Psychol. 2000;68:64-72.

47. McKay JR, Alterman AI, Cacciola JS, O'Brien CP, Koppenhaver JM, Shepard DS. Continuing care for cocaine dependence: Comprehensive 2-year outcomes. J Consult Clin Psychol. 1999;67(3):420-7.

48. Rounsaville BJ, Petry NM, Carroll KM. Single versus multiple drug focus in substance abuse clinical trials research. Drug Alcohol Depend. 2003;70(2):117-25.

49. Hubbard RL, Leimberger JD, Haynes L, Patkar AA, Holter J, Liepman MR, et al. Telephone enhancement of long-term engagement (TELE) in continuing care for substance abuse treatment: a NIDA clinical trials network (CTN) study. Am J Addict. 2007;16(6):495-502

50. Carter RE, Haynes LF, Back SE, Herrin AE, Brady KT, Leimberger
JD, Sonne SC, Hubbard RL, Liepman MR. Improving the transition from residential to outpatient addiction treatment: gender differences in response to supportive telephone calls. Am J Drug Alcohol Abuse. 2008;34(1):47-59.

51. Hughes JR, Riggs RL, Carpenter MJ. How helpful are drug abuse helplines? Drug Alcohol Depend. 2001;62(3):191-4.

52. Gigliotti A, Carneiro E, Ferreira M. Tratamento do Tabagismo. In: Rangé, B. Psicoterapias cognitivo-comportamentais: um diálogo com a psiquiatria. Porto Alegre: Artmed; 2001.

53. Stead LF, Lancaster T, Perera R. Telephone counselling for smoking cessation. Cochrane Database Syst Rev. 2003;(1):CD002850.

54. Galduróz JCF, Noto AR, Nappo SA, Carlini EA. I Levantamento domiciliar nacional sobre o uso de drogas psicotrópicas: estudo envolvendo as 24 maiores cidades do estado de São Paulo. São Paulo: Centro Brasileiro de Informações Sobre Drogas Psicotrópicas (CEBRID); 1999.

55. Zilberman ML, Tavares H, Blume SB, El-Guebaly N. Substance use disorders: sex difference and psychiatric comorbidities. Can J Psychiatry. 2003;48(1):5-13.

56. Ferigolo M, Rhoden CR, Gomez R, Barros HMT. Centros de atendimento da dependência química. Porto Alegre: Brasul; 2002. 